\title{
Macro and micro grouting process and the influence mechanism of cracks in soft coal seam
}

\author{
Hui $\mathrm{Yu}^{1,2} \cdot$ Housheng $\mathrm{Jia}^{2} \cdot$ Shaowei $\mathrm{Liu}^{2} \cdot{\mathrm{Zhihe} \mathrm{Liu}^{1} \cdot \mathrm{BaOyu} \mathrm{Li}^{1}}^{1}$
}

Received: 4 October 2020/Revised: 13 December 2020/Accepted: 30 December 2020/Published online: 26 January 2021

(C) The Author(s) 2021

\begin{abstract}
Grouting is an important method to reinforce soft coal roadway, and the presence of primary cracks in the coal body has an important influence on the grouting effect. With the discrete element simulation method, the grouting process of the soft coal seam was simulated. The mechanism of primary cracks on grouting was revealed, while the influence of fracture characteristics and grouting pressure on the grouting effect was analyzed. The results demonstrated that grouting in the soft coal seam involves the stages of seepage, rapid splitting, slow splitting, and stability. Due to the presence of primary cracks, the grouting diffusion radius increased significantly. Under the slurry pressure, the tensile stress concentration was formed at the crack tip, and the slurry split the coal once the splitting pressure was reached. In addition, the distribution characteristics of fractures are found to have a great influence on the grouting effect. It is observed that smaller fracture spacing is associated with a larger slurry diffusion radius and thus easier penetration of the primary crack tips. The fracture angle affects the direction of fracture propagation. The secondary fracture formed by splitting is a tensile fracture, which is more likely to extend along the direction parallel to the maximum principal stress. Overall, these simulation results have guiding significance for the setting of reasonable spacing of grouting holes in the practice of grouting engineering.
\end{abstract}

Keywords Discrete element simulation · Soft coal seam · Primary crack · Split grouting

\section{Introduction}

Deformation control of deep soft coal roadways is one of the greatest challenges in the mining industry (Liu et al. 2018; Jiang et al. 2019, 2020; Zuo et al. 2019). For some soft roadways, the grouting method can be used to reinforce the roadways in order to maintain their stability (Zhang et al. 2018; Stille et al. 2012; Xu et al. 2019). Currently, in an attempt to establish a theoretical model of grouting, researchers have developed the spherical

Hui Yu

yuhuizyyx@163.com

1 School of Resources and Environmental Engineering, Shandong University of Technology,

Zibo 255000, Shandong, China

2 School of Energy Science and Engineering, Henan Polytechnic University, Jiaozuo 454000, Henan, China diffusion theory, cylindrical diffusion theory, carol theory, Baker formula, and Lombardi formula, among others (Lombardi and Deere 1993; Liu et al. 2019; Xiao et al. 2019; Chen et al. 2014). A large number of engineering studies have reported that the splitting grouting process is very complicated and is the result of fluid-solid coupling between the seepage field and stress field (Zhang and Shimada 2018; Rafi and Stille 2014; Wang et al. 2019; Zhang et al. 2017; Liu and Sun 2019; Sha et al. 2019). The diffusion form of grout in coal seam cracks is difficult to obtain through laboratory tests or field measurements. The particle flow simulation method has been applied in many studies conducted in China and abroad (Sun et al.2010; Geng et al.2012; Wang et al. 2020; Zhang et al. 2018; Lei et al. 2016; Damjanac and Cundall 2016; Boschi et al. 2020; Koyama et al. 2013; Bandyopadhyay et al. 2020). This method can reveal the mechanical law and coupling mechanism of the grouting process from a microscopic perspective, reflect the relationship between the 
macroscopic performance of grouting and the microscopic characteristics of the coal body, and simulate the crack expansion under different grouting pressures.

However, the coal seam has various joints, cracks and other structural planes, which have an important impact on the mechanical properties of the coal seam. On the one hand, cracks in the coal seam can serve as slurry flow channels to reduce slurry diffusion resistance. On the other hand, because of the weakening effect of cracks on the strength of coal, the coal is more likely to split under grouting pressure, which is conducive to slurry diffusion (Gothall and Stille 2009; Jin et al. 2019; Jiang et al. 2018; Deng et al. 2018; Xiao et al. 2017). Therefore, cracks in the coal seam and their distribution characteristics have an important influence on the grouting effect. At present, the research on grouting engineering has mainly focused on the influence of grouting pressure, permeability coefficient, and other parameters on the grouting effect, while the influence mechanism of primary cracks in the coal mass on grouting has not been widely discussed (Kvartsberg and Fransson 2013; Cheng et al. 2018; Bezuijen et al.2011; Ajamzadeh et al. 2019; Hu et al.2019). In the present study, through the discrete element simulation method, the grouting process in soft coal seam was studied by microscopic simulation. Furthermore, the influence of crack characteristics and grouting pressure on grouting effect was analyzed, and the action mechanism of the primary crack on grouting was revealed.

\section{Meso-model of grouting in soft coal seam}

\subsection{Engineering-geological characteristics of soft coal seam}

At present, a mine in Xinzheng city in Henan Province, China, is mainly mining No. 21 coal seam. The coal at this mine is soft, can be easily weathered and disintegrated, and presents a loose form, with extremely low bearing capacity and an average compressive strength of approximately $0.8 \mathrm{MPa}$. The coal seam has a simple structure and belongs to the lean coal category.

The transport lane from working face 12209 in No. 21 coal seam revealed the SF19 normal fault (drop, $0-14 \mathrm{~m}$ ). The fault obliquely intersects with the working face roadway along the strike SE87 degree, which is the main fault structure affecting the working face. There are many secondary structures nearby that are influenced by faults, which make the primary cracks in the coal body more developed, as shown in Fig. 1. In order to prevent damage to the roadway due to dynamic pressure during mining, grouting reinforcement is applied to the whole section of the enlarged roadway.
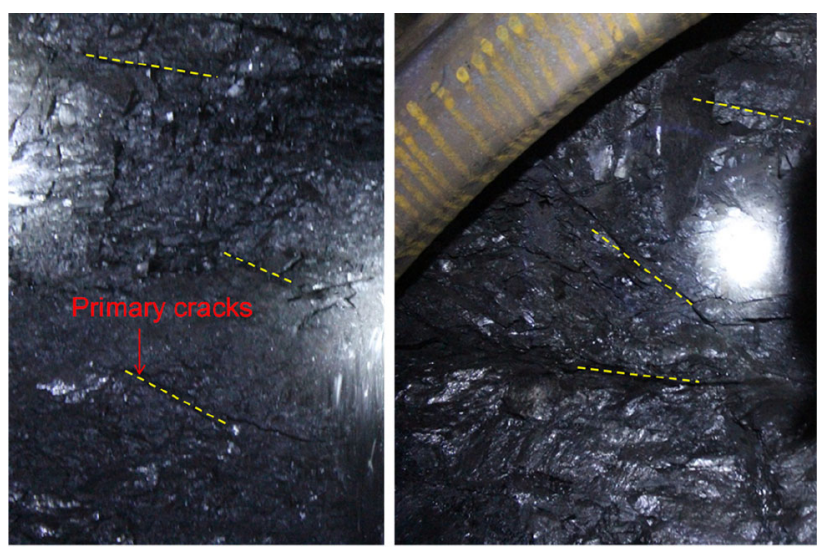

Fig. 1 Coal bodies with primary cracks

\subsection{Particle flow simulation theory of coal seam grouting}

Grouting in the coal body is a complicated coupling process between the slurry and coal body. The particle flow method was used to analyze the stress of the meso element. Whether the meso element was damaged or not was assessed according to the fracture criterion of the meso element. If the meso element is damaged, fractures will be generated. The propagation of micro-fractures forms the flow channel of the slurry. Thus, the grouting mechanism can be clarified according to the micro-level.

\subsubsection{Basic principle of fluid-solid coupling}

The theory of fluid-solid coupling of particles is based on specific basic assumptions, that is, the injected medium is simulated by the aggregate of particles. The interaction and movement of particles follow Newton's law of motion; however, there is no real fluid in the medium, which is simulated by the "fluid field" of storage pressure. The "fluid domain" consists of a series of closed particle chains, in which the stored pressure acts as an equivalent body force on the surrounding particles. The movement of simulated fluid in the injected medium is realized by a "pipeline", which is a parallel gap built on the tangent position of the particles. Its internal space is then determined by the normal displacement of the contact point of particles, and is considered as the position for simulating fluid flow. According to the particle flow method, the fluidsolid coupling is realized by the pressure stored in the fluid domain acting on the particles on both sides through the "pipe", resulting in the relative movement of particles. The size of the "pipe" changes due to the alteration in the normal displacement between particles, which then leads to the change of the "fluid domain" volume, thus realizing the fluid-solid interaction process. In Fig. 2, the red dots 


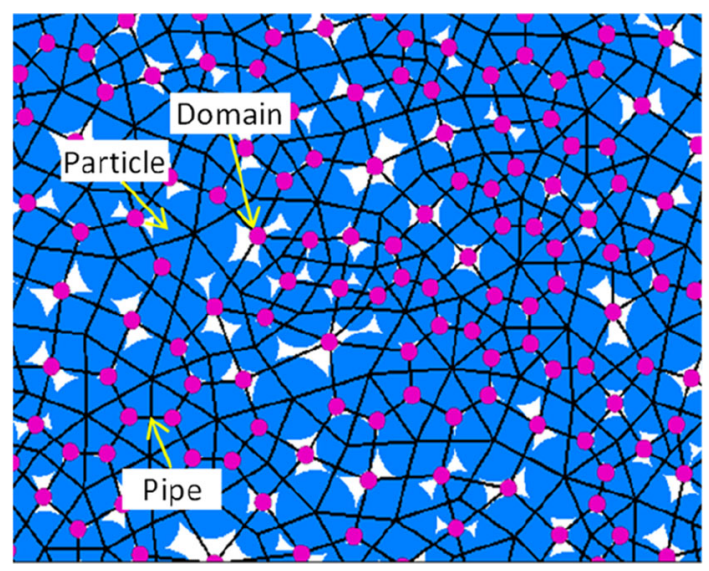

Fig. 2 Fluid domain and particle coupling

represent the domain, the black lines of the connecting domain represent the "pipes" for fluid flow, and the blue circles represent the coal particles.

\subsubsection{Flow and pressure equations}

Fluid is stored in the pore grid, and fluid exchange can occur under the action of fluid pressure difference in adjacent pore grids. In order to quantitatively calculate the flow rate during fluid exchange, it can be assumed that the fluid channel is a parallel plate channel at the contact point of two adjacent particles. The flow rate between the two pores can be expressed according to the Hagen-Poiseuille equation, as follows:

$q=k a^{3} \frac{\Delta p}{L}$

where, $q$ is the flow rate in $\mathrm{m}^{3} / \mathrm{s}, a$ represents the opening of the fluid passage and is related to the normal force of the two particles, $k$ is the permeability coefficient, $\Delta p$ is the pressure difference between the two pore grids, and $L$ is the fluid passage length.

In the $\Delta t$ time step, the pore fluid pressure change caused by fluid flow is calculated by the volume compression modulus of the fluid. A certain pore is considered to have $\mathrm{n}$ fluid channels. In the $\Delta t$ time step, the total fluid flow is $\sum q$, and the change in the pore fluid pressure is calculated as follows:

$\Delta p=\frac{K_{\mathrm{f}}}{V_{\mathrm{d}}}\left(\sum q \Delta t-\Delta V_{\mathrm{d}}\right)$

where, $K_{\mathrm{f}}$ represents the compression modulus of the fluid, and $V_{\mathrm{d}}$ is the pore volume.

\subsection{Establishment of grouting model for soft coal seam}

In the particle flow simulation, the mechanical parameters used in the model are the micro-mechanical parameters of particles. Different micro-mechanical parameters of particles have a different effect on the macro-mechanical properties of the coal body. By continuously adjusting the micro-mechanical parameters of the particle body and contact, simulation results that are close to the real macromechanical properties of the coal body can be obtained. In the present study, laboratory tests were firstly carried out on coal samples obtained from the transportation lane of the working face 12209 in order to obtain the macroscopic physical and mechanical parameters of the soft coal body. Next, simulation tests were conducted multiple times to obtain the microscopic parameters of the particle aggregate, consistent with the macroscopic parameters, as shown in Tables 1 and 2. According to the particle flow method, the real fluid is simulated by the "fluid domain" of storage pressure. By performing several Darcy seepage experiments, the basic parameters of the fluid domain were obtained, as shown in Table 3.

Subsequently, in order to analyze the influence of primary cracks on the grouting diffusion characteristics of the coal mass, models were respectively established for the cases of absence or presence of primary cracks. The diffusion process of coal seam grouting was explored from the macro and micro perspectives, and the influence mechanism of coal seam primary cracks on grouting was determined. In the actual coal body, the crack dip angle and crack spacing are not represented by a single value, which are usually in line with the normal distribution. However, in order to facilitate the quantitative analysis of the influence of fracture distribution on the slurry diffusion, the primary cracks were arranged regularly, as shown in Fig. 3.

In Case I, there were no primary cracks in the coal body. By contrast, in Case II, primary cracks were present in the coal body, with a crack spacing $(D)$ of $0.5 \mathrm{~m}$ and a crack angle $(\alpha)$ of $60^{\circ}$. The width $\times$ height of the two models was $2 \mathrm{~m} \times 2 \mathrm{~m}$, and the grouting pressure of the grouting hole was $1.5 \mathrm{MPa}$. The boundary of the calculation model is set as impermeable boundary. The smooth joint model was used to simulate the primary cracks of the coal body, and the parameters were shown in Table 4. A parallel bond model was adopted to explore the contact between the particles, which could not only simulate the normal and tangential forces between the coal particles, but also simulate the rotation and bending moment between these particles. 
Table 1 Macroscopic parameters of coal particles

\begin{tabular}{lllll}
\hline Modulus of elasticity $E(\mathrm{GPa})$ & Internal friction angle $\varphi\left(^{\circ}\right)$ & Cohesion $C(\mathrm{MPa})$ & Poisson's ratio $\lambda$ & Density $\left(\mathrm{g} / \mathrm{cm}^{3}\right)$ \\
\hline 2.2 & 34 & 1.3 & 0.25 & 1.8 \\
\hline
\end{tabular}

Table 2 Mesoscopic parameters of coal particles

\begin{tabular}{lllllll}
\hline $\begin{array}{l}\text { Particle minimum } \\
\text { radius } R_{\min }(\mathrm{m})\end{array}$ & $\begin{array}{l}\text { Ratio of particle } \\
\text { radius } R_{\max } / R_{\min }\end{array}$ & $\begin{array}{l}\text { Friction } \\
\text { coefficient }\end{array}$ & $\begin{array}{l}\text { Normal bond } \\
\text { strength }(\mathrm{MPa})\end{array}$ & $\begin{array}{l}\text { Tangential bond } \\
\text { strength }(\mathrm{MPa})\end{array}$ & $\begin{array}{l}\text { Normal contact } \\
\text { stiffness } k_{\mathrm{n}}(\mathrm{MN} / \mathrm{m})\end{array}$ & $\begin{array}{l}\text { Particle } \\
\text { stiffness ratio } \\
k_{\mathrm{n}} / k_{\mathrm{s}}\end{array}$ \\
\hline 0.01 & 1.5 & 0.4 & 0.3 & 0.3 & 100 & 1.5 \\
\hline
\end{tabular}

Table 3 Basic parameters of fluid domain

\begin{tabular}{lllll}
\hline $\begin{array}{l}\text { Apparent volume of domain } \\
V_{\mathrm{d}}\left(\mathrm{mm}^{3}\right)\end{array}$ & $\begin{array}{l}\text { Number of pipes in a } \\
\text { domain } N\end{array}$ & $\begin{array}{l}\text { Pipe diameter } \\
a(\mathrm{~mm})\end{array}$ & $\begin{array}{l}\text { Fluid bulk modulus } K_{\mathrm{f}} \\
(\mathrm{GPa})\end{array}$ & $\begin{array}{l}\text { Permeability coefficient } \\
k(\mathrm{~cm} / \mathrm{s})\end{array}$ \\
\hline 1 & 2 & 1 & 1 & $\begin{array}{l}\text { Time step } \\
\Delta t(\mathrm{~s})\end{array}$ \\
\hline
\end{tabular}

\section{Results of grouting simulation of soft coal seam}

\subsection{Analysis of grouting process}

The splitting grouting diffusion process is shown in Fig. 4 under a grouting pressure of $1.5 \mathrm{MPa}$ and with the increase in grouting time. The red line segments in figures indicate that the bonds between particles are broken and that splitting cracks are formed by coal grouting. Black circles 1,2 and 3 represent monitoring circles with a radius of $0.25,0.5$ and $0.75 \mathrm{~m}$, respectively. Each monitoring circle was set to monitor the evolution law of porosity and coal stress in the circle during grouting and was used as a measure of the slurry diffusion radius. Figure 5 shows the evolution of the internal porosity of the coal body with time for the two cases investigated, with the dotted line representing Case I and the solid line representing Case II.

As shown in Figs. 4 and 5, with the increase in grouting time, the distribution range of cracks in the coal seam gradually increased, and the slurry diffusion process can be divided into the following three stages:

(1) Slurry infiltration stage (step $=100)$ : In the initial stage, the grouting pressure had a compaction impact on the coal medium surrounding the grouting hole and resulted in the plastic damages in coal (Fig. 4a). However, the slurry could not split the coal medium over this stage and the fracture damages are mainly gathering around the pipe hole. As observed in Fig. 4, the porosity of the coal mass slightly increased, and the diffusion radius of the slurry reached $0.15 \mathrm{~m}$.

(2) Rapid splitting stage of slurry (step $=100-1000$ ): With further progression of the grouting process, the splitting effect was generated when the splitting pressure was reached in the coal seam (Fig. 4b). The slurry flowed rapidly along the splitting surface, and the porosity increased rapidly. when the new generated cracks in Case II connected with its primary cracks, the slurry diffusion radius had a signification difference between two cases that the maximum radius in Case I reached $0.3 \mathrm{~m}$ over 500 steps and the radius in Case II increased from $0.5 \mathrm{~m}$ to $0.7 \mathrm{~m}$ after the connection of the new and primary cracks.

(3) Slow splitting-stabilization stage (step more than 1000): The slurry flowed and diffused along the fracture under a certain grouting pressure gradient. After the rapid grouting stage, the splitting effect on the coal seam was weakened as the pressure of the slurry that was far away from the grouting hole gradually decreased, and finally the fracture expansion was relatively stable and no longer developing to the deep part. The maximum diffusion radius of the slurry was $0.8 \mathrm{~m}$ in Case II, while the porosity in the monitoring circle slightly increased at this stage.

As shown in Fig. 5, the grouting porosity under the conditions of Case II was larger than that in Case I, indicating that more crack channels were generated under Case II. Monitoring circle 1 had the largest porosity, indicating that more cracks were produced closer to the grouting hole. 


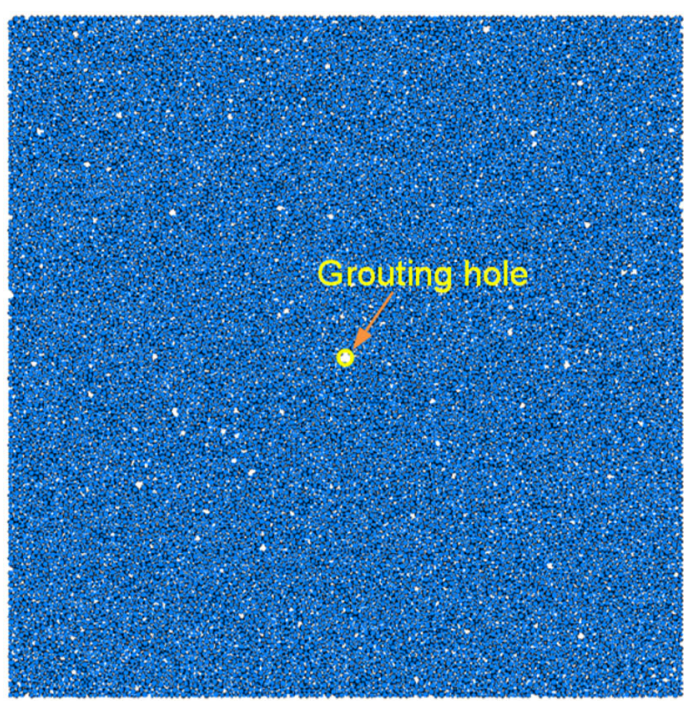

(a) Case I

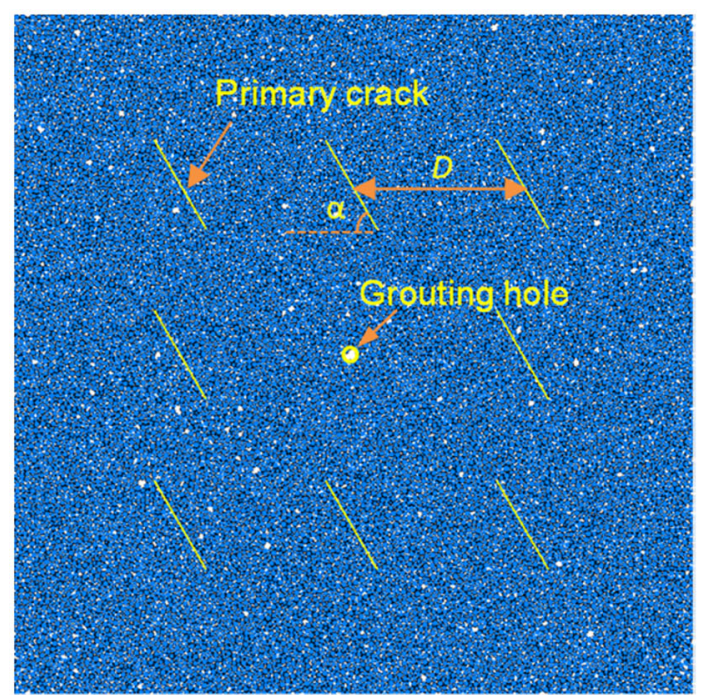

(b) Case II

Fig. 3 Discrete element model of grouting in soft coal seam

With the splitting flow of slurry, the porosity changed from rapid growth to slow growth, and finally reached stability.

The evolution of the contact force inside the coal during grouting is displayed in Fig. 6. The color of the lines in the figure represents the magnitude of the contact force. The contact force inside the coal body can reveal the compression effect of grout pressure on the coal body and reflect the distribution characteristics of the grout pressure.
Furthermore, the white area in the figure represents the crack channel generated by grouting. Due to the squeezing effect of slurry in the crack on the coal body, the contact force between the cracks was relatively high. As shown in Fig. 6, the grouting pressure range gradually expanded outward from the grouting hole, while the highest pressure was observed around the grouting hole, decreasing gradually along the radial direction. As the grouting time increased, the maximum contact force also gradually increased and finally tended to be stable. The maximum contact force in Case I was increased from 30.6 to $50.6 \mathrm{kN}$.

In Case II, on the side of the primary crack facing away from the grouting hole, the contact force was evidently reduced, and a weak contact force zone appeared. The main contact force was distributed radially (Fig. 6d), which indicates that the void generated by the primary crack damaged the integrity of the coal body and hindered the transmission of the contact force.

According to the abovementioned observations, it was evident that when the coal seam contained more primary cracks, the slurry diffusion characteristics were quite different from those of the whole coal body. In Case II, with the increase in grouting time, the secondary fractures generated by coal seam splitting were connected with the primary cracks. The sequence number in Case II displayed in Fig. 4d represents the sequence of the fracture generation. The penetration position (1) (Fig. 4d) was always located at the crack tip. Stress concentration was easily produced at the crack tip, resulting in easier splitting. Due to the small flow resistance of the slurry in the crack, the slurry would flow along the primary cracks and continue to split the coal seam at the other tip of the cracks. The diffusive radius of grouting markedly increased in the presence of primary cracks, increasing from $0.65 \mathrm{~m}$ to more than $0.76 \mathrm{~m}$ compared with Case I.

\subsection{Stress field change of grouting coal}

The stress field of coal body is the macro performance of the contact force of particles. The average stress $\bar{\sigma}$ in a measurement region of volume $V$ is computed as (Christoffersen et al. 1981):

$\bar{\sigma}=-\frac{1}{V} \sum_{N_{\mathrm{c}}} F^{(\mathrm{c})} \otimes L^{(\mathrm{c})}$

Table 4 Parameters of smooth joint model

\begin{tabular}{llllll}
\hline Normal stiffness $(\mathrm{MN} / \mathrm{m})$ & Shear stiffness $(\mathrm{MN} / \mathrm{m})$ & Friction coefficient & Cohesion $(\mathrm{MPa})$ & Tensile strength (MPa) & Crack apertures (mm) \\
\hline 2000 & 2000 & 0.35 & 0 & 0 & 1 \\
\hline
\end{tabular}



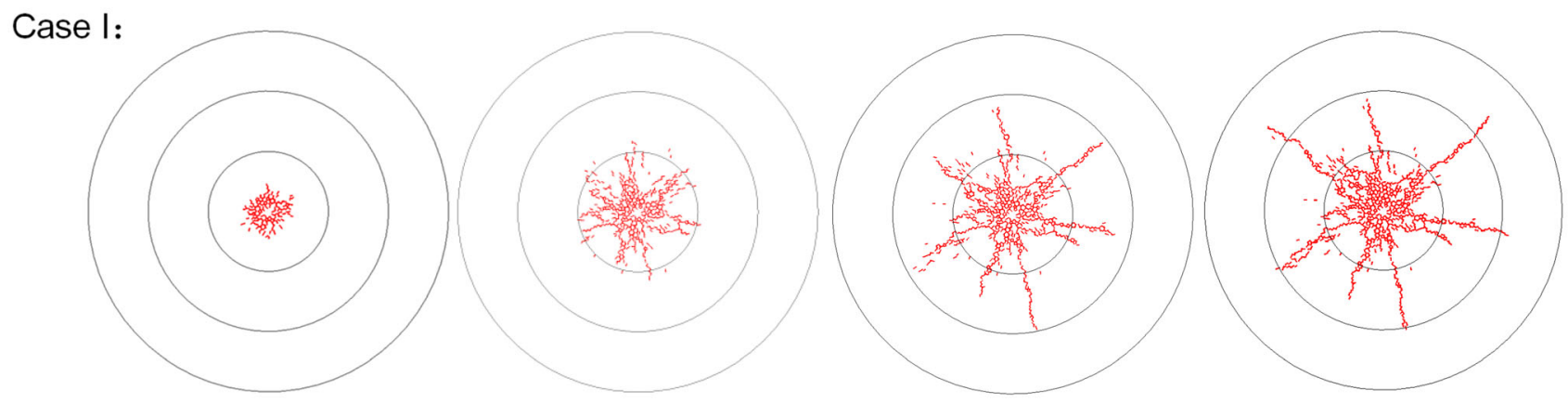

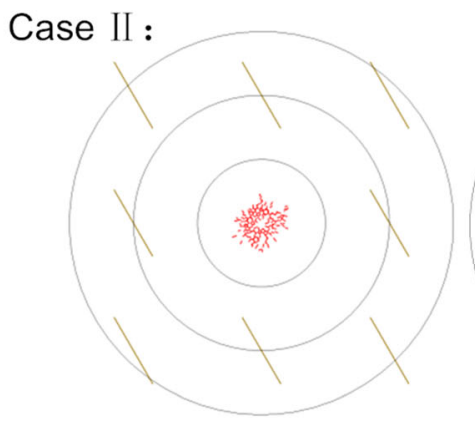

(a) Step $=100$

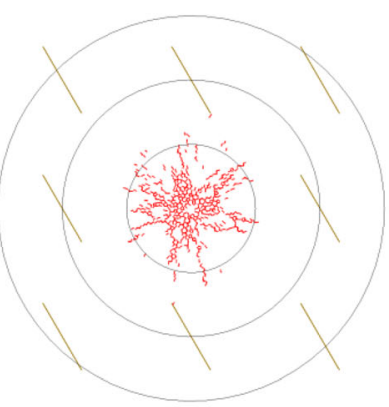

(b) Step $=500$

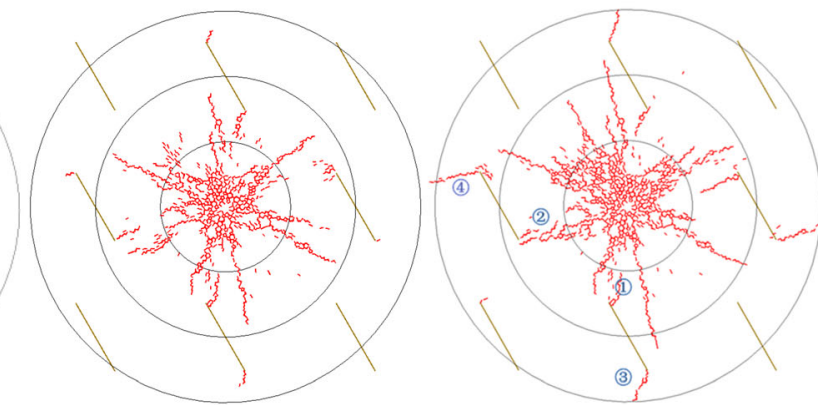

(c) Step $=1000$ (d) Step $=3000$

Fig. 4 Diffusion process of grouting in soft coal seam

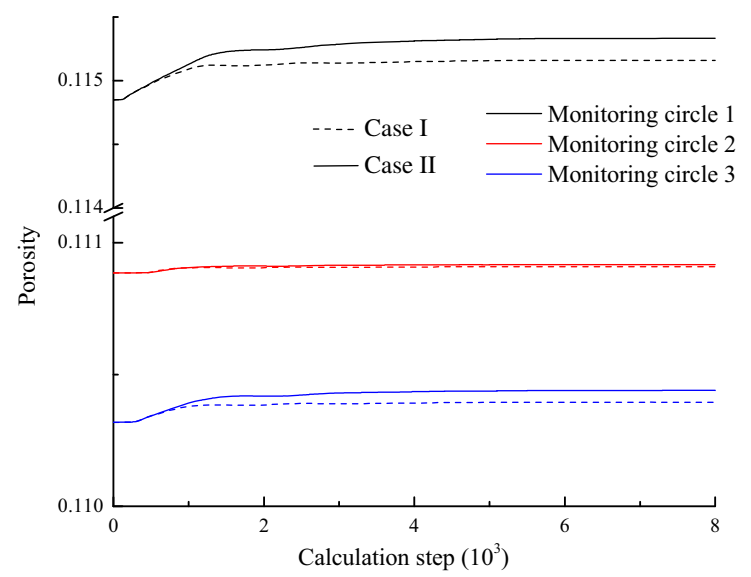

Fig. 5 Porosity evolution process in different monitoring circles

where $N_{\mathrm{c}}$ is the number of contacts that lie in the measurement region or on its boundary, $F^{(c)}$ is the contact force vector, $L^{(\mathrm{c})}$ is the branch vector joining the centroids of the two bodies in contact, $\otimes$ denotes outer product, and compressive stress is negative by convention.

Stress curves for the three monitoring circles during grouting are shown in Fig. 7. It can be seen from the figure that the normal stress and shear stress of Case II were higher than those of Case I under the same conditions, which indicates that grouting was more effective in Case II. Among the three monitoring circles, the value of normal stress was the highest for monitoring circle 1, indicating that the closer to the grouting hole, the denser the slurry distribution, and the greater the compressive stress on the coal body. There are three distinct stages of stress change in grouting process, including the rapid stress rising stage (0-2000 steps), slow stress rising stage (2000-4000 steps) and stress stabilization stage ( $>4000$ steps).

During the period of rapid stress rise, the slurry splitting fracture expanded rapidly, which is considered as the main stage of the grouting process. The stress between particles increased sharply, with more intense stress value changes were observed closer to the grouting hole. With the development of a split fracture to the deep coal body, the pressure of the slurry in the fracture gradually decreased. The splitting effect on the coal body was also gradually weakened, and the stress was slowly raised. Finally, when the slurry hydraulic pressure gradually decreased to less than the splitting pressure, the stress between coal particles did not change with time, and the grouting process tended to be stable. When comparing the normal stress and shear stress curves, it can be observed that the normal pressure was much larger than the shear stress, indicating that the compression deformation of coal particles was mainly caused by mutual extrusion during the grouting process, while the slip and stagger deformation of particles was relatively small. 

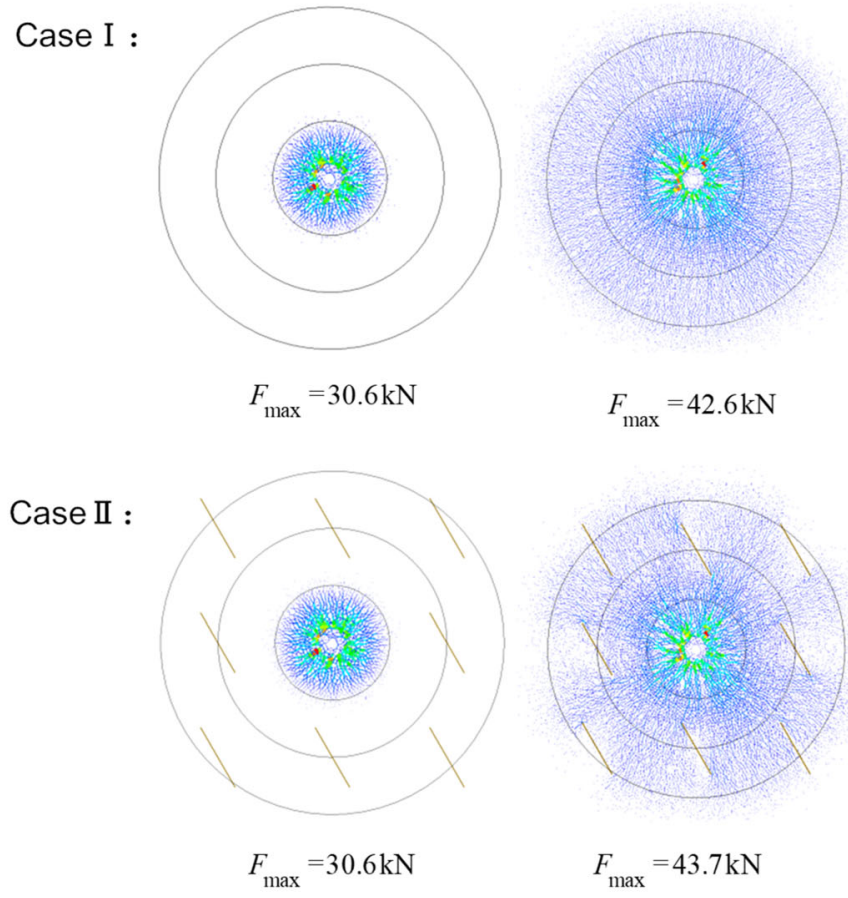

(a) Step $=100$

(b) $\mathrm{Step}=500$

Fig. 6 Evolution of particle contact force in the coal body

\subsection{Strain rate change of grouting coal}

The $x$-direction strain rate curve of the coal particles in different monitoring ranges is presented in Fig. 8. In this figure, the change of the strain rate of coal reflects the speed of grouting. The variation trend of the strain rate of the coal body in different monitoring ranges shown in the figure was basically the same. In the same monitoring range, the strain rate of Case II was higher than that of Case I, indicating that grouting in Case II was more effective. During the initial stage of grouting, the strain rate was high, slurry splitting and rapid diffusion were observed, after which the strain rate decreased gradually, indicating that the slurry diffusion rate slowed down gradually and finally tended to be stable. The maximum strain rate in the monitoring circle 1 was 0.54 , while the maximum strain rate in monitoring circle 3 was only 0.06 , which indicated that the slurry diffusion gradually decreased from the grouting hole to the surrounding area.

\subsection{Analysis of the crack mechanism of grouting in the coal seam}

The formation of grouting fractures is due to the splitting force of grouting fluid on the coal body. In order to study the splitting of the primary crack tip under grouting, the stress at the tip was monitored by investigating points A-D
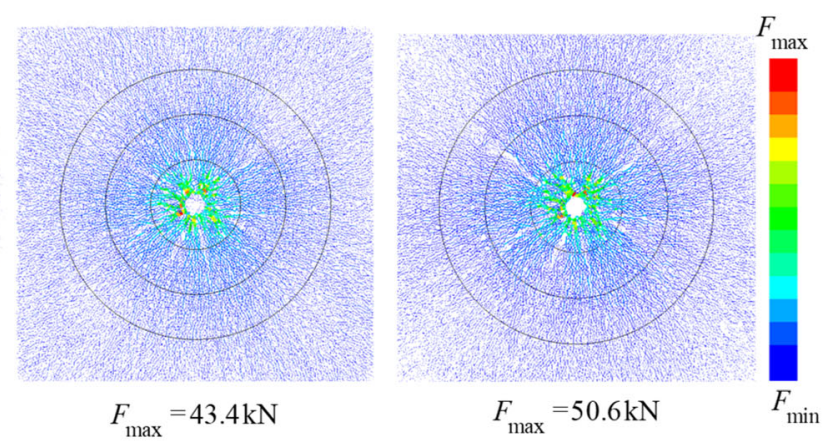

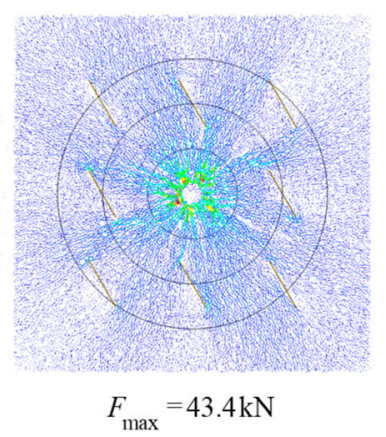

(c) Step $=1000$

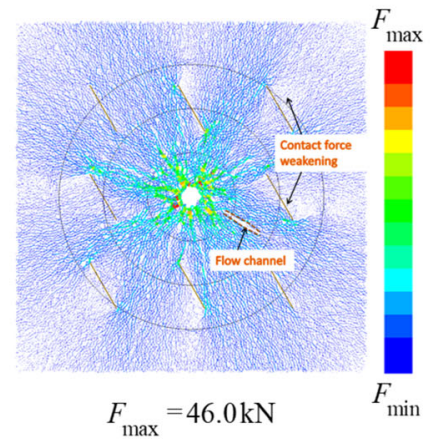

(d) Step $=3000$ that are shown in Fig. 9. The radius of the points is $0.03 \mathrm{~m}$. The internal contact force at step $=500$ is displayed in Fig. 9a, where the blue line represents the compressive stress, and the brown line represents the tensile stress. In addition, the internal tensile stress at step $=2000$ is shown in Fig. 9b. Since the fracture propagation is mainly associated with the tensile stress, only the distribution characteristics of the tensile stress are shown. At the periphery of the grouting hole, a tensile stress ring was formed, which surrounded the crack generated by the grouting. At the newly formed secondary crack at the tip of the primary crack, tensile stress concentration was also generated. Therefore, the tensile stress at the tip of the crack is considered as the driving force for further expansion of the crack.

The stress evolution curve at the fracture tip is presented in Fig. 10, where negative values represent the tensile stress. The A and B tip curves represent the $y$-direction stress, while the $\mathrm{C}$ and $\mathrm{D}$ tip curves represent the $x$-direction stress. According to the graph, the maximum tensile stress at the crack tip $\mathrm{C}$ was higher than that at the $\mathrm{D}$ point, which indicates that the energy of the slurry was gradually weakened after flowing through the primary crack, and its ability in splitting the coal seam was further weakened. When the maximum tensile stress was less than the tensile strength between particles, the coal seam could not be further split by the slurry, and thus the grouting stopped. 


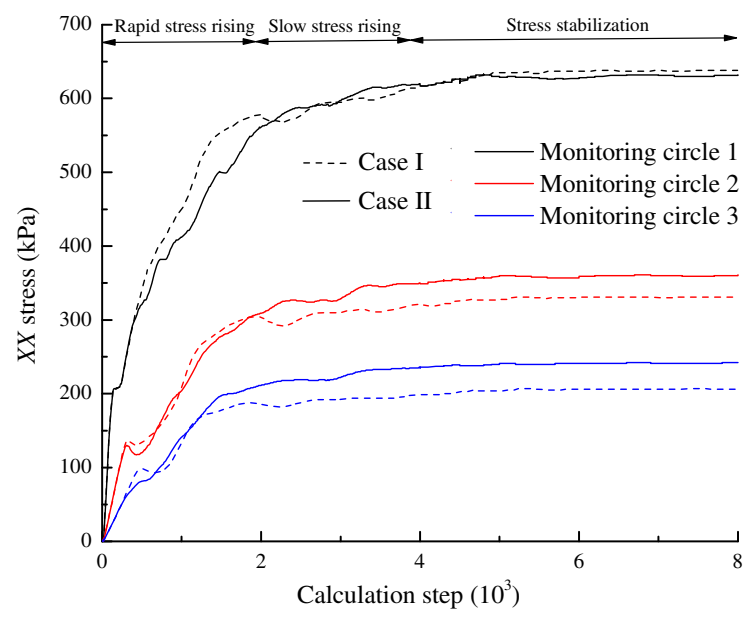

(a) $X$-direction normal stress

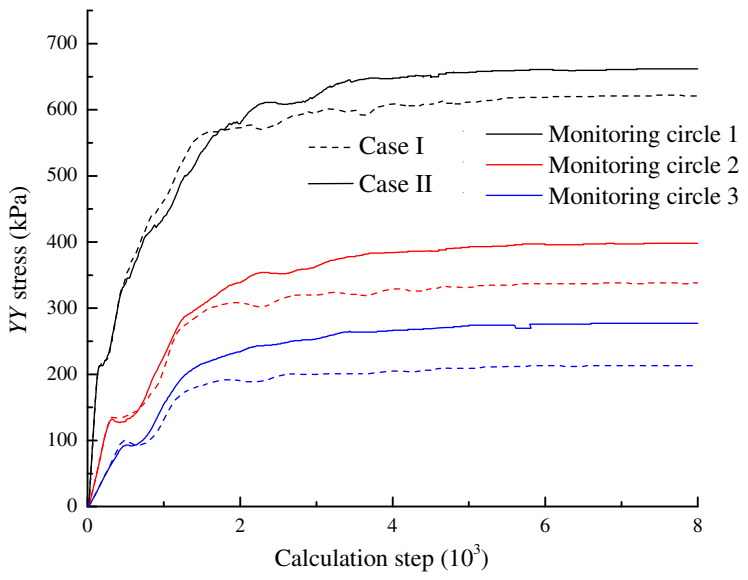

(b) $Y$-direction normal stress

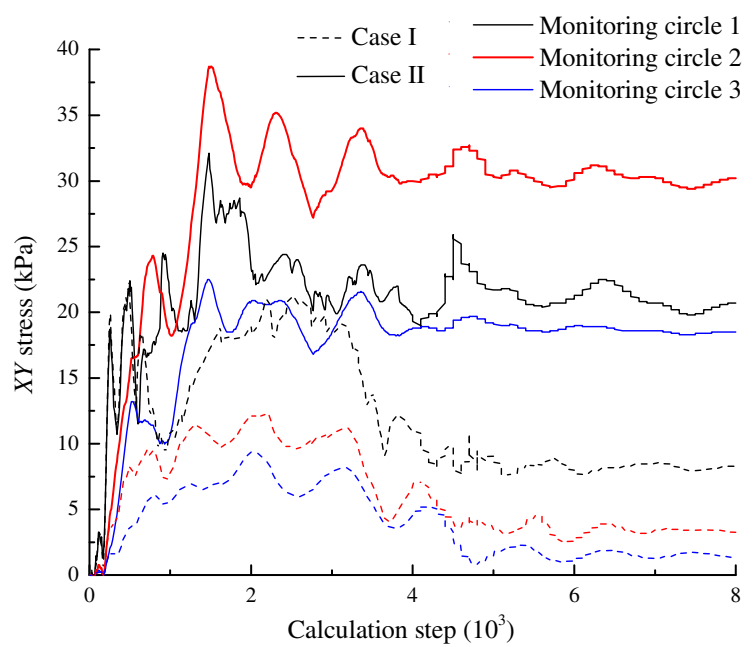

(c) $X Y$ shear stress

Fig. 7 Stress evolution curve of the coal body

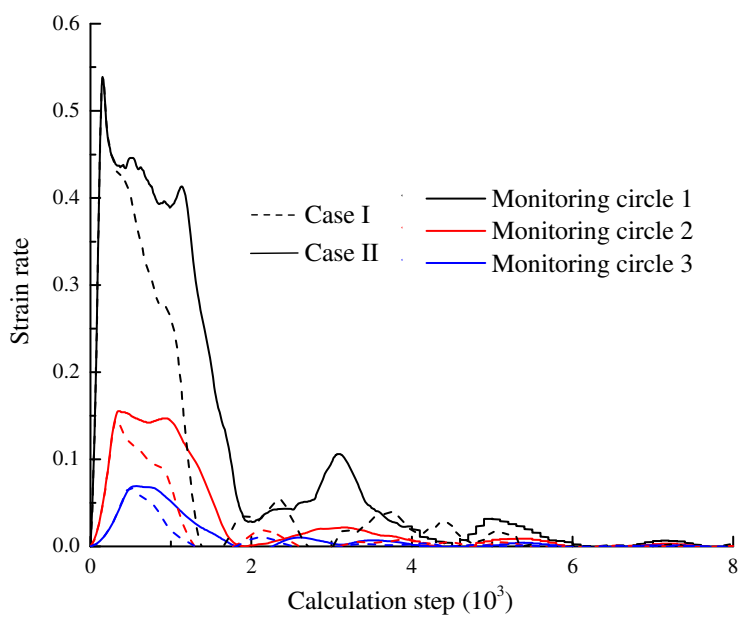

Fig. 8 Strain rate evolution curve of the coal body

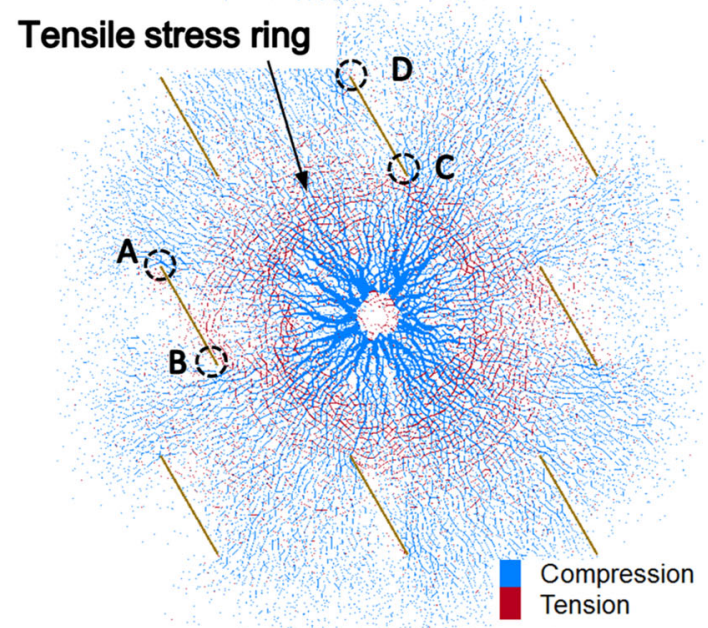

(a) Contact force chain distribution at step $=500$

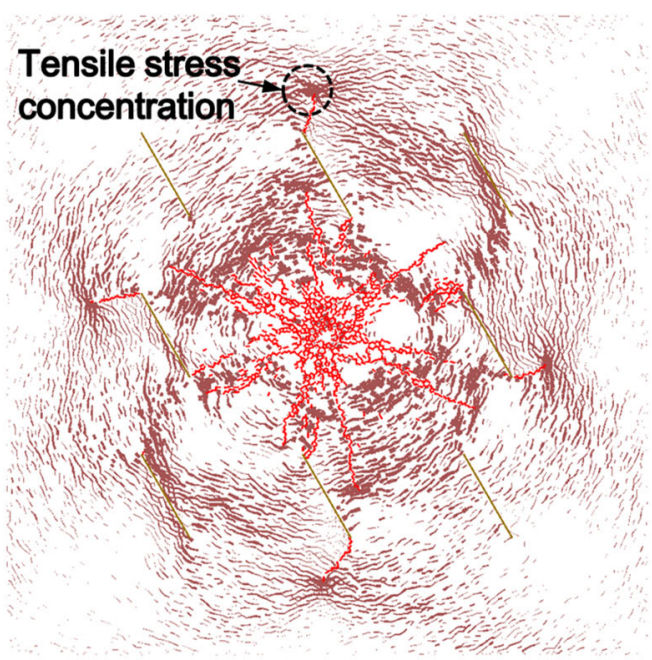

(b) Tensile stress distribution at step $=2000$

Fig. 9 Internal stress distribution of the coal body 


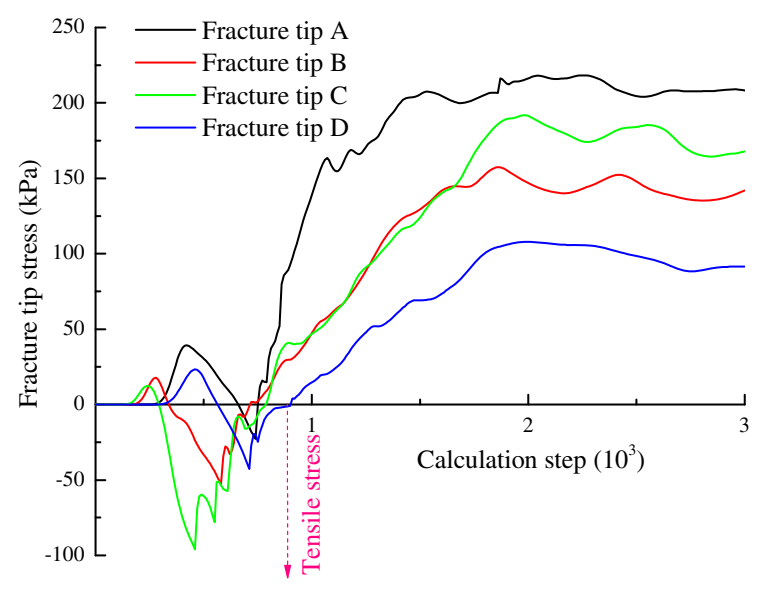

Fig. 10 Stress evolution curve at the crack tip

\section{Influence of grouting pressure and crack distribution on grouting}

The grouting process is affected by multiple factors. In engineering practice, different grouting effects can be obtained by applying different grouting pressures. At the same time, in multi-jointed coal seams, the fracture distribution characteristics will also affect the slurry diffusion path.

\subsection{Influence of grouting pressure on grouting effect}

The slurry diffusion characteristics under different grouting pressures are presented in Fig. 11. It can be seen that a larger grouting pressure resulted in a more dense fracture distribution. At a value of $P=1 \mathrm{MPa}$, the radius of the influence range of coal grouting was about $0.5 \mathrm{~m}$, and the coal particles outside this range were basically not affected by grouting. At a value of $P=1.5 \mathrm{MPa}$, the radius of the influence area of coal grouting evidently increased, reaching $0.7 \mathrm{~m}$. Furthermore, at $P=2 \mathrm{MPa}$, the radius of the influence of grouting hardly increased, whereas the fractures produced by grouting increased greatly. This observation indicates that the grouting diffusion was more effective at $2 \mathrm{MPa}$ compared with other pressures, whereas the damage to the surrounding coal body was also greater. Therefore, in actual grouting engineering, there is a reasonable grouting pressure that does not only ensure the influence range of grouting, but also does not destroy the bearing capacity of the surrounding coal.

Furthermore, the $x$-direction stress distribution of coal under different grouting pressures is displayed in Fig. 12. A higher grouting pressure increased the internal stress of coal and resulted in a larger influence range in the coal body.

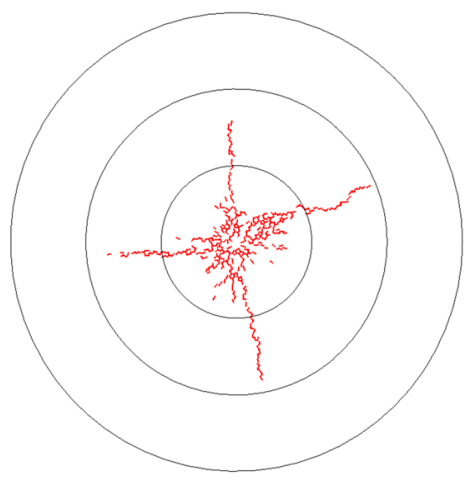

(a) $P=1.0 \mathrm{MPa}$

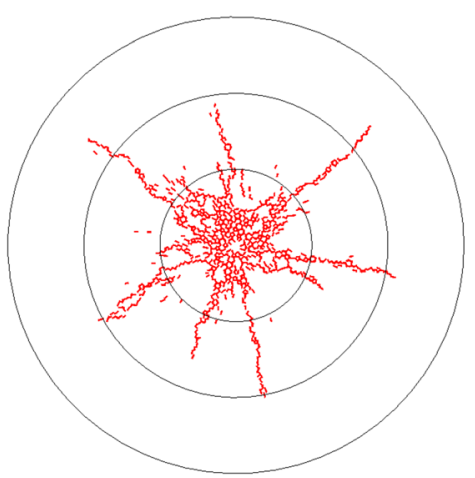

(b) $P=1.5 \mathrm{MPa}$

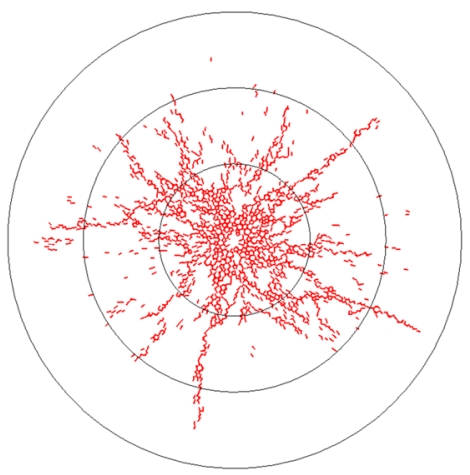

(c) $P=2.0 \mathrm{MPa}$

Fig. 11 Slurry diffusion range under different grouting pressure

\subsection{Influence of crack distribution characteristics on the grouting effect}

The geometric characteristics of multi-cracks mainly include the distribution and arrangement of cracks. The following section mainly explores the influence of crack spacing and angle on the slurry diffusion characteristics.

\subsubsection{Crack spacing}

Crack spacing represent the degree of crack development in coal, which is an important index for evaluating the flow 


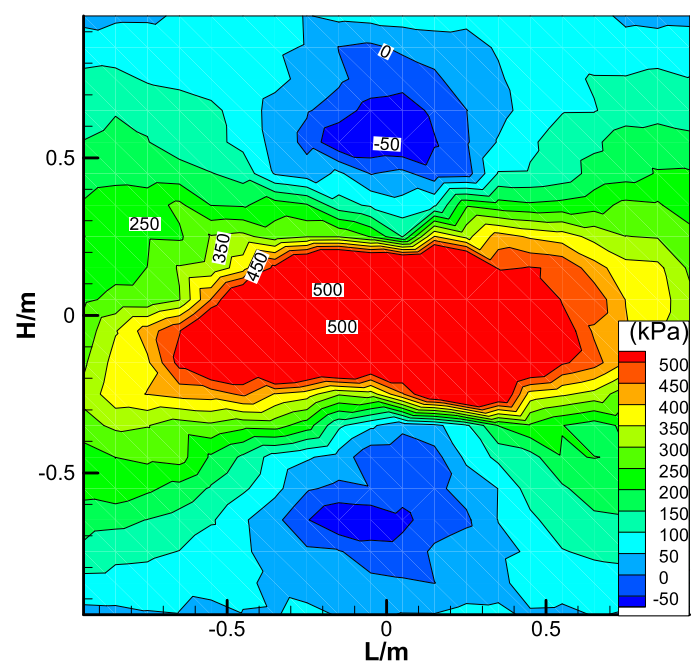

(a) $P=1.0 \mathrm{MPa}$

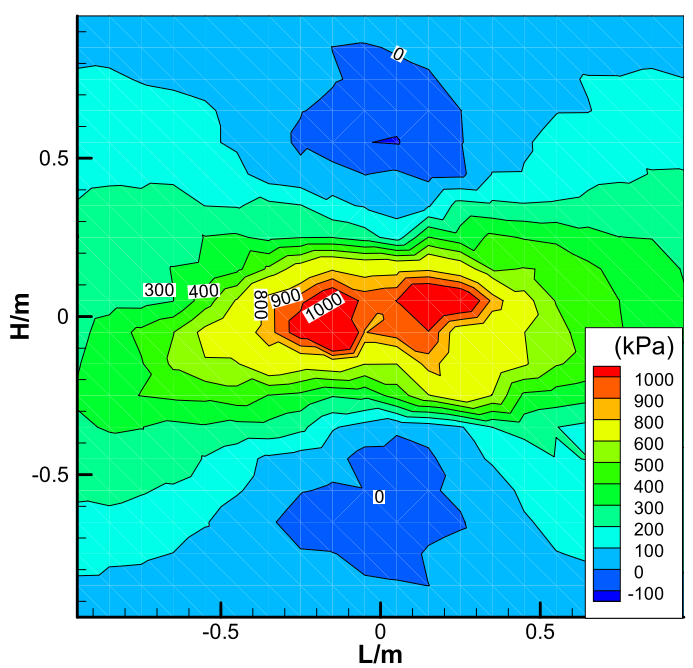

(b) $P=1.5 \mathrm{MPa}$

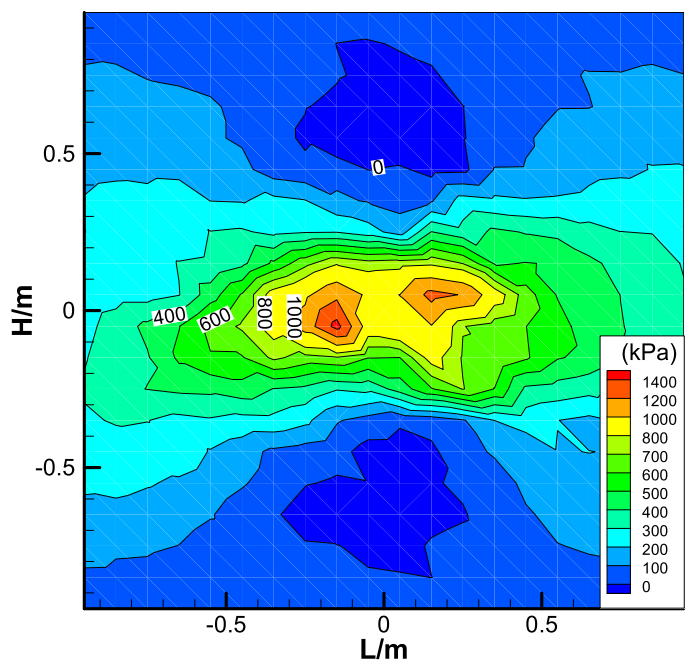

(c) $P=2.0 \mathrm{MPa}$
4Fig. $12 X$-direction stress distribution of coal under different grouting pressure

capacity of cracked coal. The horizontal distance between two adjacent crack planes of the same crack group is defined as the crack spacing, and equal crack spacing is observed in the same crack group.

The slurry diffusion characteristics at different crack spacing under the condition of $P=1.5 \mathrm{MPa}$ and $\alpha=60^{\circ}$, including at $0.3,0.5$, and $0.7 \mathrm{~m}$, respectively, are shown in Fig. 13. With the decrease of crack spacing, a greater number of crack channels produced by grouting were associated with a larger diffusion radius. At crack spacing

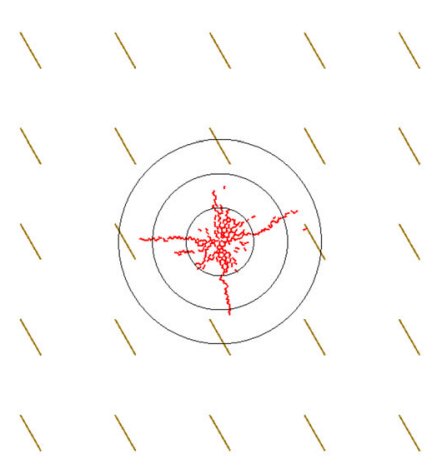

(a) $D=0.7 \mathrm{~m}$

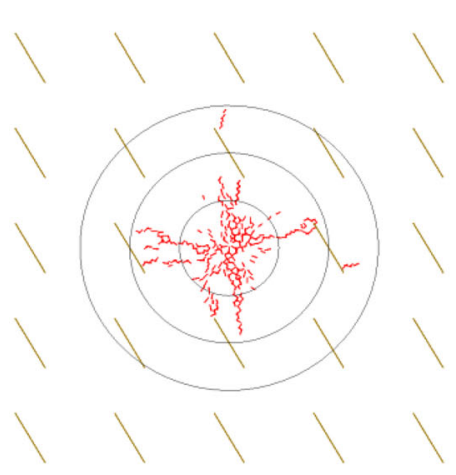

(b) $D=0.5 \mathrm{~m}$

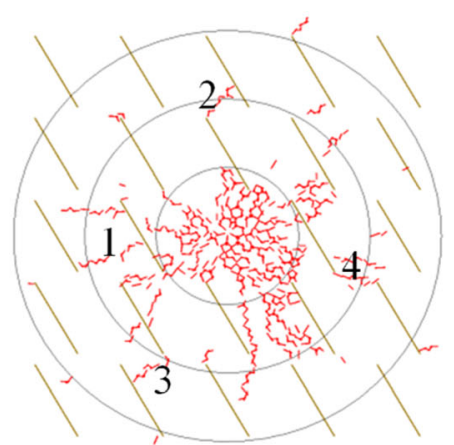

(c) $D=0.3 \mathrm{~m}$

Fig. 13 Slurry diffusion range with different crack spacing 
$D=0.7 \mathrm{~m}$, the fractures produced by the slurry could not connect with the primary cracks, and the primary cracks had no obvious influence on the grouting radius, which is equivalent to Case I. At $D=0.5 \mathrm{~m}$, the grout diffusion radius had already spread to the primary crack, and the generated fracture was connected with the primary crack. The grout flowed along the primary crack and continued to diffuse outward, while the grouting radius increased to $0.75 \mathrm{~m}$. Furthermore, at crack spacing $D=0.3 \mathrm{~m}$, the splitting fractures produced by grouting were further increased, and the intersection occurred between the primary cracks, as shown in Fig. 13c, which further extended the grouting channel. Therefore, when the crack spacing is small, the fractures produced by the slurry will connect the primary cracks of the coal body and further increase the diffusion radius by grouting.

\subsubsection{Crack angle}

The slurry diffusion characteristics of different crack angle under the condition of $P=1.5 \mathrm{MPa}$ and $D=0.5 \mathrm{~m}$, including $\alpha=0^{\circ}, 30^{\circ}$, and $60^{\circ}$, respectively, are shown in Fig. 14.As it can be seen from Fig. 14, under the same grouting pressure, the crack angle had little effect on the grouting radius; however, it had a great influence on the crack propagation path and direction. As shown in Fig. $14 \mathrm{a}$, for the primary crack a, the slurry continued to flow forward after flowing through the primary crack when $\alpha=0^{\circ}$, resulting in fracture 1 , which expanded in the same direction as that of the fracture a. The nearest position between primary crack $b$ and the grouting hole was in the middle of crack $b$. Some slurry was able to penetrate the fracture near the middle of crack $b$ and to extend along the direction perpendicular to the primary crack, resulting in fracture 2. The remaining part of the slurry flowed along crack b and split at the tip to form fracture 3 .

As shown in Fig. 14b, c, the slurry flowed along the primary crack and split at the tip. However, the splitting fracture did not follow the direction of the primary crack, and was at an angle with the primary crack, which was related to the maximum principal stress at the tip. Since the secondary fracture formed by splitting was a tensile fracture, it was more likely to propagate along the direction parallel to the maximum principal stress.

\section{Grouting project of soft coal seam}

The roadway 12209 is located in the No. 21 coal seam. It is supported by a full bolt-mesh and constructed along the roof of the coal seam. The original cross-section of roadway is $16 \mathrm{~m}^{2}$, and the total cross-section of the enlarged roadway is $27.9 \mathrm{~m}^{2}$. In order to prevent the roadway from

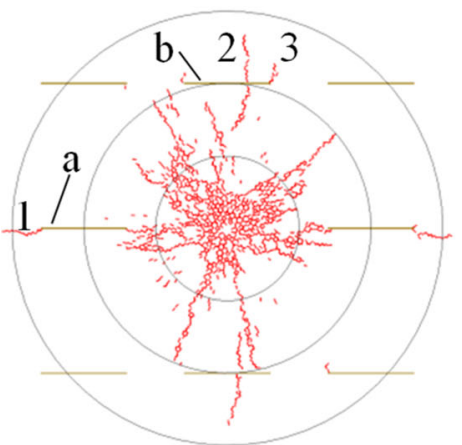

(a) $\alpha=0^{\circ}$

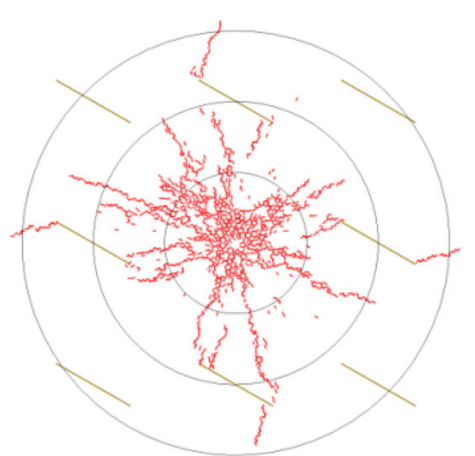

(b) $\alpha=30^{\circ}$

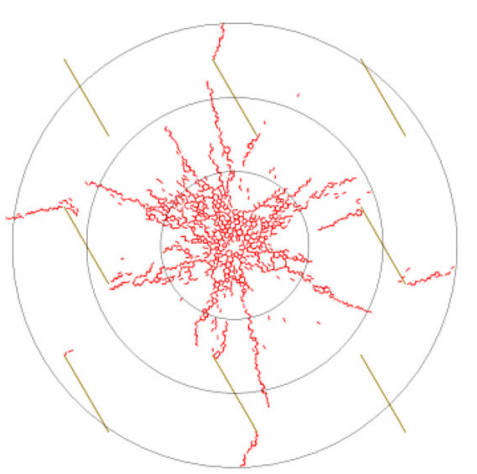

(c) $\alpha=60^{\circ}$

Fig. 14 Slurry diffusion range with different crack angle

being damaged by dynamic pressure during mining, grouting reinforcement is applied to the entire section of the enlarged roadway.

Grouting holes were created by an anchor rope drill. The slurry was mixed with a vertical mixer, and the ZBY-80/ 7.0 two-liquid grouting pump was used for grouting. Considering the performance of the grouting equipment and according to the field construction experience, the simulation values of grouting pressure were 1.0, 1.5, and 2.0 MPa, respectively. The change curve of the slurry diffusion radius with grouting time under different grouting pressures is shown in Fig. 15. As seen in the figure, with the increase in grouting time, the growth rate of the slurry diffusion radius gradually decreased, while the effect of 


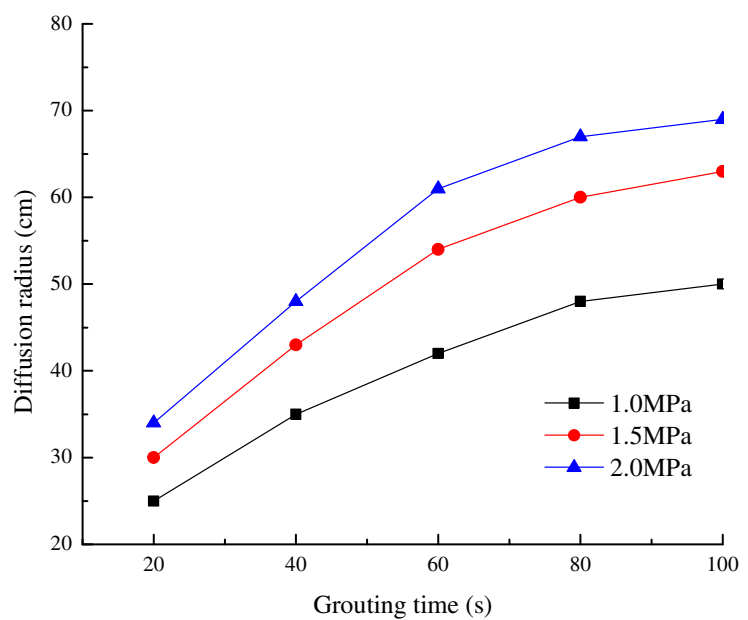

Fig. 15 Variation curve of slurry diffusion radius under different grouting pressures

high grouting pressure on the increase of slurry diffusion radius was limited; therefore, the final grouting hole pressure was set to $1.5 \mathrm{MPa}$.

According to the simulation results, the diffusion radius can reach $0.63 \mathrm{~m}$ when the grouting pressure is $1.5 \mathrm{MPa}$ in the case of coal without primary cracks. Since there are many cracks in the coal seam of this roadway, the actual diffusion radius is larger. Therefore, in order to ensure the grouting effect, as well as take into account the technical and economic factors, the grouting hole row spacing was set to $1.6 \mathrm{~m}$, and each row had the same hole depth. A total of eleven grouting holes were arranged in each row, with four holes at the top, one at each of the two sides, and five at the bottom, as shown in Fig. 16. The grouting sequence involved grouting from bottom to top. Single liquid cement slurry was used as the grouting material, and $52.5 \mathrm{R}$ ordinary Portland cement was mixed with water at a ratio of 1:0.75.

After full-section grouting, the soft coal was reinforced. Under a grouting pressure of $1.5 \mathrm{MPa}$, the slurry diffused well, and the maximum diffusive radius reached approximately $0.8 \mathrm{~m}$. This shows that it is feasible to determine the diffusive radius of soft coal under a certain grouting pressure by simulation, while the reasonable row distance of grouting holes can also be obtained accordingly. After grouting, the deformation of the roadway was significantly reduced, and the stability of the roadway in the soft coal seam was effectively improved.

\section{Conclusions}

(1) In the present study, it was reported that the grouting process of the soft coal seam involved the stages of seepage, rapid splitting, slow splitting, and then

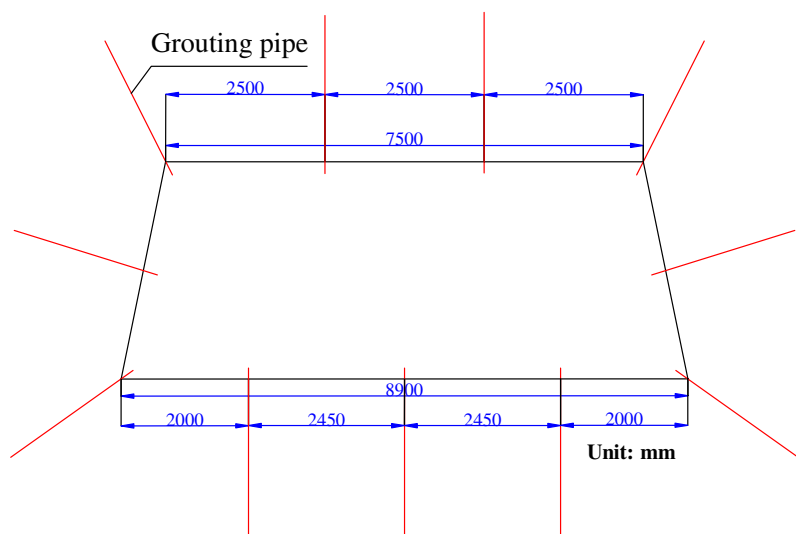

(a) Full section of the roadway

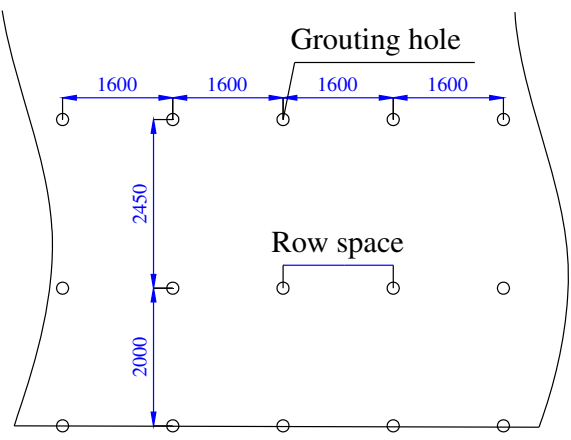

(b) Grouting hole of the floor

Fig. 16 Layout of grouting holes in roadway 12209

stability. The existence of primary cracks in the coal body had an important influence on the grouting effect of internal stress and fracture propagation during the grouting process. Secondary fractures produced by splitting penetrated the primary cracks of the coal seam. The slurry flowed along the primary cracks and continued to split the coal seam at the other tip of the cracks. The existence of primary cracks significantly increased the grouting diffusion radius, while it also increased the stress level of the coal body, resulting in more adequate grouting.

(2) During grouting, the fracture propagation was mainly caused by the tensile stress at the crack tip. Due to the effect of the slurry pressure, tensile stress concentration was formed at the crack tip, leading to coal seam splitting by the grouting fluid. The slurry pressure gradually decreased from the grouting hole to the grouting hole, and thus the tensile stress at the tip was also gradually decreased. Finally, the grout diffusion tended to be stable.

(3) The characteristics of the crack distribution had a great influence on the grouting effect. It was observed that smaller fracture spacing was 
associated with a larger slurry diffusion radius and thus easier penetration of the primary crack tips. The crack angle affected the direction of fracture propagation. Furthermore, the secondary fracture formed by splitting was a tensile fracture. When the grouting pressure was small, the grouting radius increased along with the increase in pressure. However, when the grouting pressure reached a certain value, increasing the grouting pressure had little effect on the grouting radius.

\section{Discussion}

The results of the present study suggest that, according to the discrete element simulation, the grout diffusion range under a certain grouting pressure can be obtained, which has great guiding significance for setting a reasonable grouting hole spacing in grouting engineering practice. Due to the irregular crack distribution in the real coal body, there is inevitable deviation between the actual diffusion radius of the slurry and the simulation value. Therefore, attention should be paid to the actual crack development of the coal body during the grouting construction.

Acknowledgments The authors acknowledge the financial support provided by the National Natural Science Foundation of China (No. 51604094 and 51674098), and the Shandong Provincial Natural Science Foundation (No.ZR2020QE118).

Data availability The data used to support the findings of this study are available from the corresponding author upon request.

\section{Compliance with ethical standards}

Conflict of interest The authors declare that they have no conflicts of interest.

Open Access This article is licensed under a Creative Commons Attribution 4.0 International License, which permits use, sharing, adaptation, distribution and reproduction in any medium or format, as long as you give appropriate credit to the original author(s) and the source, provide a link to the Creative Commons licence, and indicate if changes were made. The images or other third party material in this article are included in the article's Creative Commons licence, unless indicated otherwise in a credit line to the material. If material is not included in the article's Creative Commons licence and your intended use is not permitted by statutory regulation or exceeds the permitted use, you will need to obtain permission directly from the copyright holder. To view a copy of this licence, visit http://creativecommons. org/licenses/by/4.0/.

\section{References}

Ajamzadeh M, Sarfarazi V, Dehghani H (2019) Evaluation of plow system performance in long-wall mining method using particle flow code. Int J Coal Sci Technol 6(4):518-535
Bandyopadhyay K, Mallik J, Ghosh T (2020) Dependence of fluid flow on cleat aperture distribution and aperture-length scaling: a case study from Gondwana coal seams of Raniganj Formation, Eastern India. Int J Coal Sci Technol 7(1):133-146

Bezuijen A, Grotenhuis R, Tol AF (2011) Analytical model for fracture grouting in sand. $\mathrm{J}$ Geotech geoenviron Eng 137(6):611-620

Boschi K, Prisco CG, Ciantia MO (2020) Micromechanical investigation of grouting in soils. Int J Solids Struct 187:121-132

Chen TL, Zhang LY, Zhang DL (2014) An FEM/VOF hybrid formulation for fracture grouting modelling. Comput Geotech 58:14-27

Cheng WC, Ni JC, Shen JS (2018) Modeling of permeation and fracturing grouting in sand: laboratory investigations. J Test Eval 46(5):2067-2082

Christoffersen J, Mehrabadi MM, Nemat-Nasser S (1981) A micromechanical description of granular material behavior. J Appl Mech 48:339-344

Damjanac B, Cundall P (2016) Application of distinct element methods to simulation of hydraulic fracturing in naturally fractured reservoirs. Comput Geotech 71(1):283-294

Deng SH, Wang XL, Yu J (2018) Simulation of grouting process in rock masses under a dam foundation characterized by a $3 \mathrm{~d}$ fracture network. Rock Mech Rock Eng 51:1801-1822

Geng P, Lu ZK, Ding T (2012) Study on dynamic process simulation of surrounding rock grouting based on particle flow. J Rock Mech Eng 31:445-452

Gothall R, Stille H (2009) Fracture-fracture interaction during grouting. Tunn Undergr Sp Technol 25:199-204

$\mathrm{Hu}$ JH, Ren QF, Ma SW et al (2019) Macroscopic and microscopic trans-scale characteristics of pore structure of mine grouting materials. T Nonferr Metal Soc 29:1067-1081

Jiang DH, Cheng XZ, Luan HJ (2018) Experimental investigation on the law of grout diffusion in fractured porous rock mass and its application. Processes 6(10):191

Jiang LS, Kong P, Shu JM et al (2019) Numerical Analysis of Support Designs Based on a Case Study of a Longwall Entry. Rock Mech Rock Eng 52(9):3373-3384

Jiang LS, Kong P, Zhang PP et al (2020) Dynamic Analysis of the Rock Burst Potential of a Longwall Panel Intersecting with a Fault. Rock Mech Rock Eng 53(4):1737-1754

Jin LC, Sui WH, Xiong JL (2019) Experimental investigation on chemical grouting in a permeated fracture replica with different roughness. Appl Sci 9(13):2762

Koyama T, Katayama T, Tanaka T et al (2013) Development of a numerical model for grout injection and its application to the in situ grouting test at the Grimsel test site. Switzerland Geosyst Eng 16(1):26-36

Kvartsberg S, Fransson A (2013) Hydrogeological characterisation and stochastic modelling of a hydraulically conductive fracture system affected by grouting: A case study of horizontal circular drifts. Tunn Undergr Sp Technol 38(9):38-49

Lei QH, Latham JP, Tsang CF (2016) The use of discrete fracture networks for modelling coupled geomechanical and hydrological behaviour of fractured rocks. Comput Geotech 85:151-176

Liu QS, Sun L (2019) Simulation of coupled hydro-mechanical interactions during grouting process in fractured media based on the combined finite-discrete element method. Tunn Undergr Sp Technol 84(2):472-486

Liu SW, Fu MX, Jia HS et al (2018) Shear Characteristics of Cuneiform Reaming Anchorage Bolts in Coal Mine Roadways. Rock Mech Rock Eng 52(6):1931-1943

Liu XL, Wang F, Huang J et al (2019) Grout diffusion in silty fine sand stratum with high groundwater level for tunnel construction. Tunn Undergr Sp Technol 93(11):103051.1-103051.11 
Lombardi G, Deere D (1993) Grouting design and control using the GIN principle. Int Water Power Dam Constr 45(6):15-22

Rafi JY, Stille H (2014) Control of rock jacking considering spread of grout and grouting pressure. Tunn Undergr Sp Technol 40(2):1-15

Sha F, Lin CJ, Li ZF (2019) Reinforcement simulation of water-rich and broken rock with Portland cement-based grout. Constr Build Mater 221:292-300

Stille H, Gustafson G, Hassler L (2012) Application of new theories and technology for grouting of dams and foundations on rock. Geotech Geol Eng 30(3):603-624

Sun F, Zhang DL, Chen TL (2010) Study on meso-mechanical simulation of fracture grouting in soil. Chin J Geotech Eng 32(3):474-480

Wang XC, Liu RT, Yang WM (2019) Study on diffusion mechanism of horizontal fracture grouting considering water evolution of cement slurry. J Rock Mech Eng 35(5):1005-1017

Wang X, Yuan W, Yan YT (2020) Scale effect of mechanical properties of jointed rock mass: a numerical study based on particle flow code. Geomech Eng 21(3):259-268

Xiao F, Shang JL, Zhao ZY (2019) DDA based grouting prediction and linkage between fracture aperture distribution and grouting characteristics. Comput Geotech 112:350-369
Xiao F, Zhao ZY, Chen HM (2017) A simplified model for predicting grout flow in fracture channels. Tunn Undergr Sp Technol 70:11-18

Xu ZP, Liu CW, Zhou XW et al (2019) Full-scale physical modelling of fissure grouting in deep underground rocks. Tunn Undergr Sp Technol 89(7):249-261

Zhang DM, Gao P, Yin ZY (2017) Particle flow simulation of tunnel seepage erosion. Geotech Mech 38:429-438

Zhang ZY, Hideki S (2018) Numerical study on the effectiveness of grouting reinforcement on the large heaving floor of the deep retained goaf-side gateroad: a case study in china. Energies 11(4):1001-1015

Zhang ZL, Shao ZS, Fang XB et al (2018) Research on the fracture grouting mechanism and pfc numerical simulation in loess. Adv Mater Sci Eng 2018:1-7

Zhang DL, Sun F, Li PF (2012) Research and application of composite grouting mechanism for submarine tunnel. J Rock Mech Eng 31:445-452

Zuo JP, Wang JT, Jiang YQ (2019) Macro/meso failure behavior of surrounding rock in deep roadway and its control technology. Int J Coal Sci Technol 6(3):301-319 